\title{
Engineering the PP7 Virus Capsid as a Peptide Display Platform
}

Liangjun Zhao, ${ }^{1}$ Mykhailo Kopylov, ${ }^{3}$ Clinton S. Potter, ${ }^{3}$ Bridgett Carragher, ${ }^{3}$ M.G. Finn ${ }^{1,2}$ *

${ }^{1}$ School of Chemistry and Biochemistry, Georgia Institute of Technology, 901 Atlantic Drive, Atlanta, GA, 30332, USA

${ }^{2}$ School of Biological Sciences, Georgia Institute of Technology, 901 Atlantic Drive, Atlanta, GA, 30332, USA

${ }^{3}$ National Resource for Automated Molecular Microscopy, Simons Electron Microscopy Center, New York Structural Biology Center, 89 Convent Ave, New York, NY, USA

\section{Supporting information}

Figure S1. Characterization of the indicated proteins comprising hybrid PP7 Z-domain particles by representatifve LC-MS (left), transmission electron microscopy (TEM) of negatively-stained particles (middle), and dynamic light scattering (DLS, right).
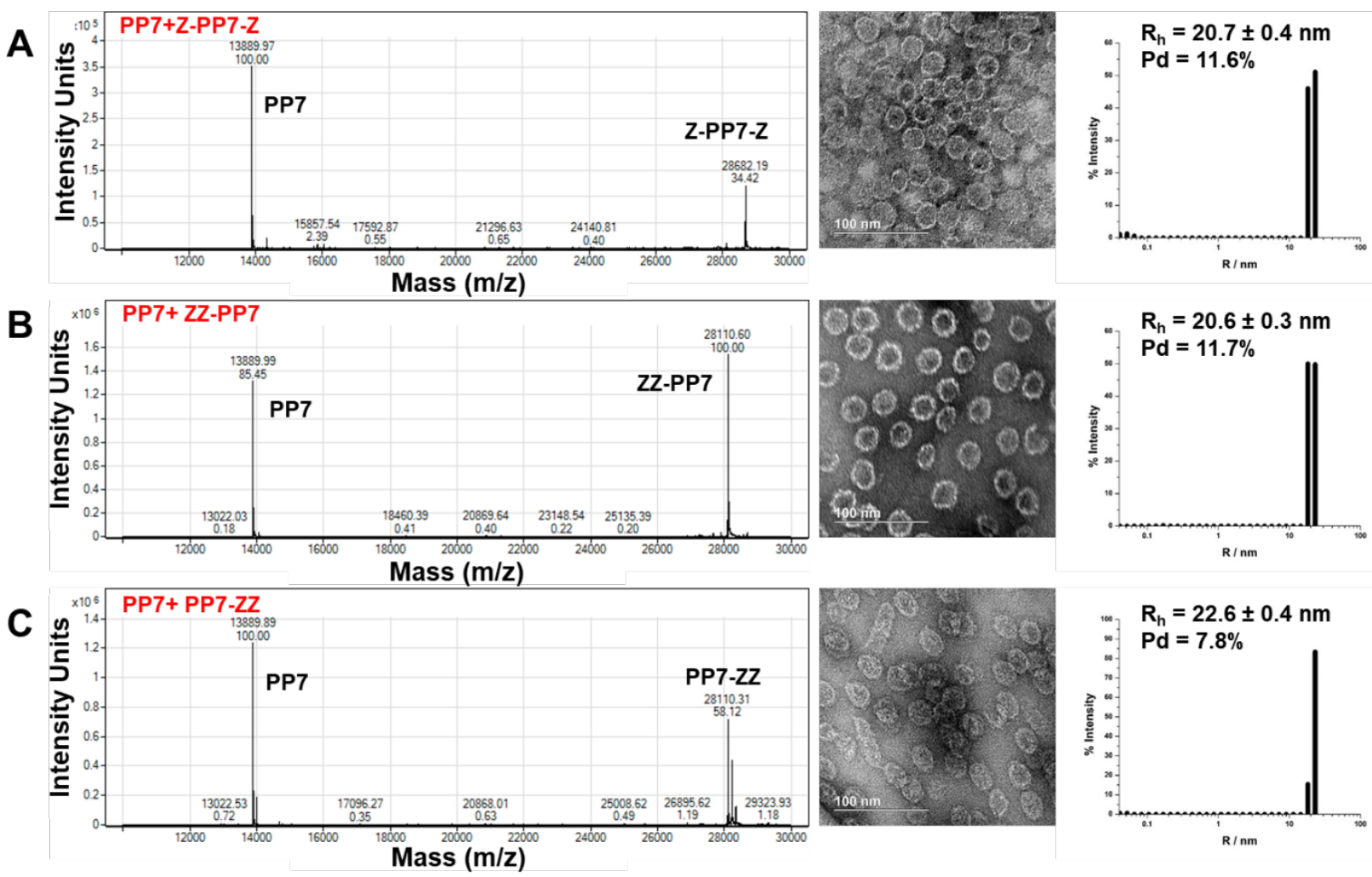
Figure S2. Characterization of the indicated proteins comprising homogeneous PP7 particles with Zdomain extensions by representative LC-MS (left), TEM of negatively-stained particles (middle), and DLS (right).
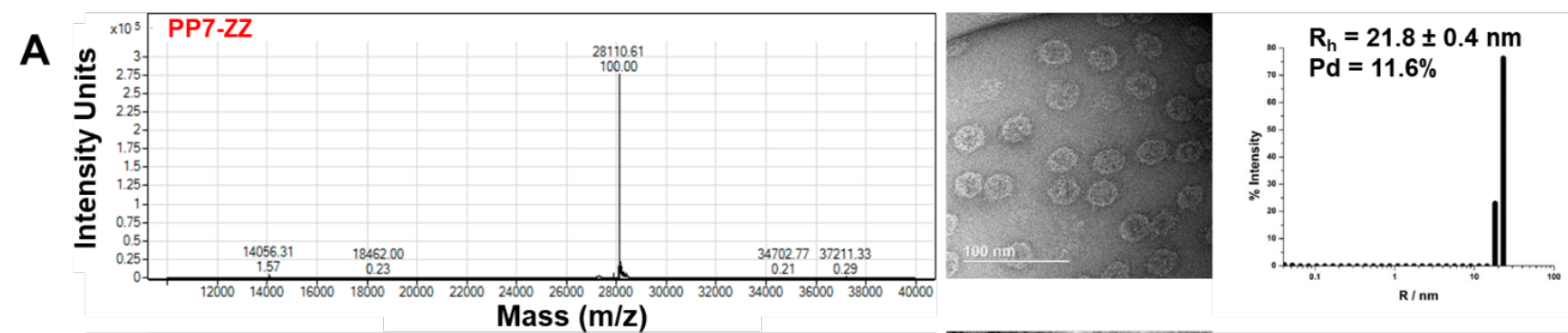

B
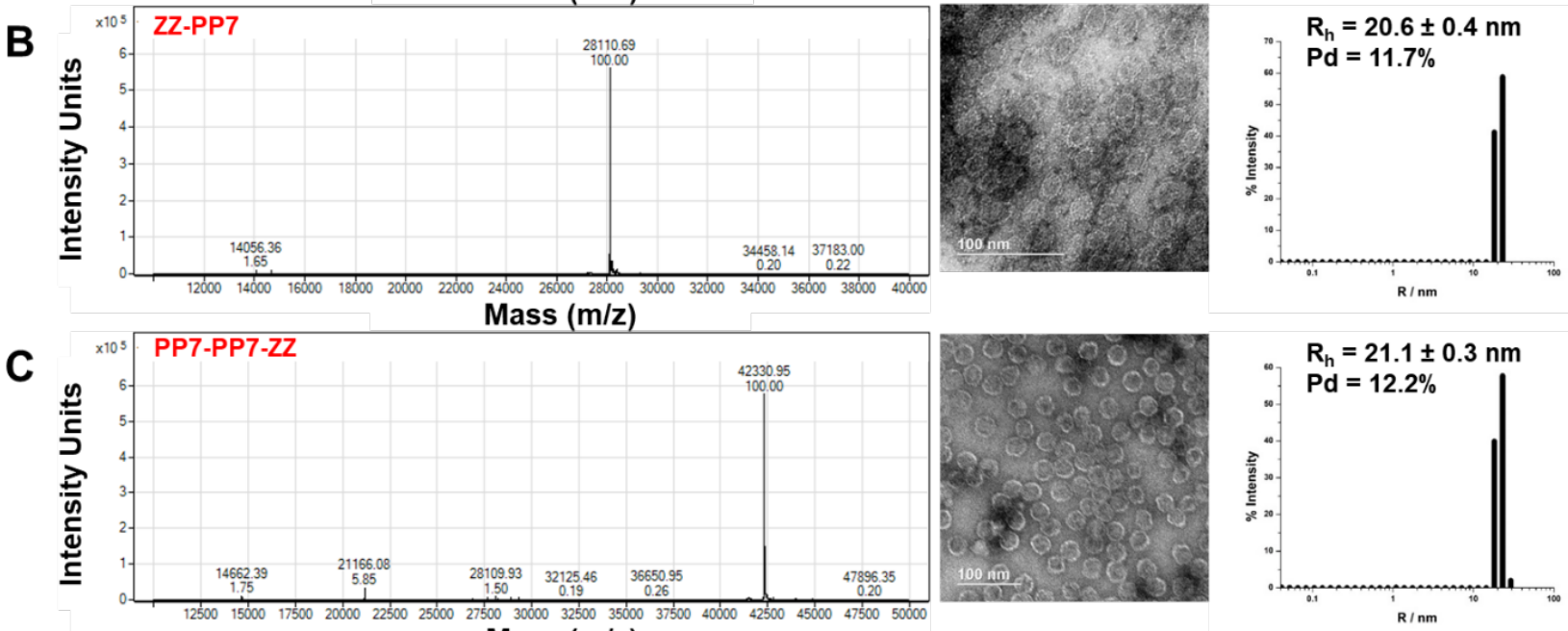
Figure S3. Characterization of the indicated proteins comprising homogeneous monomeric PP7 particles with other extensions by representative LC-MS (left), TEM of negatively-stained particles (middle), and DLS (right).
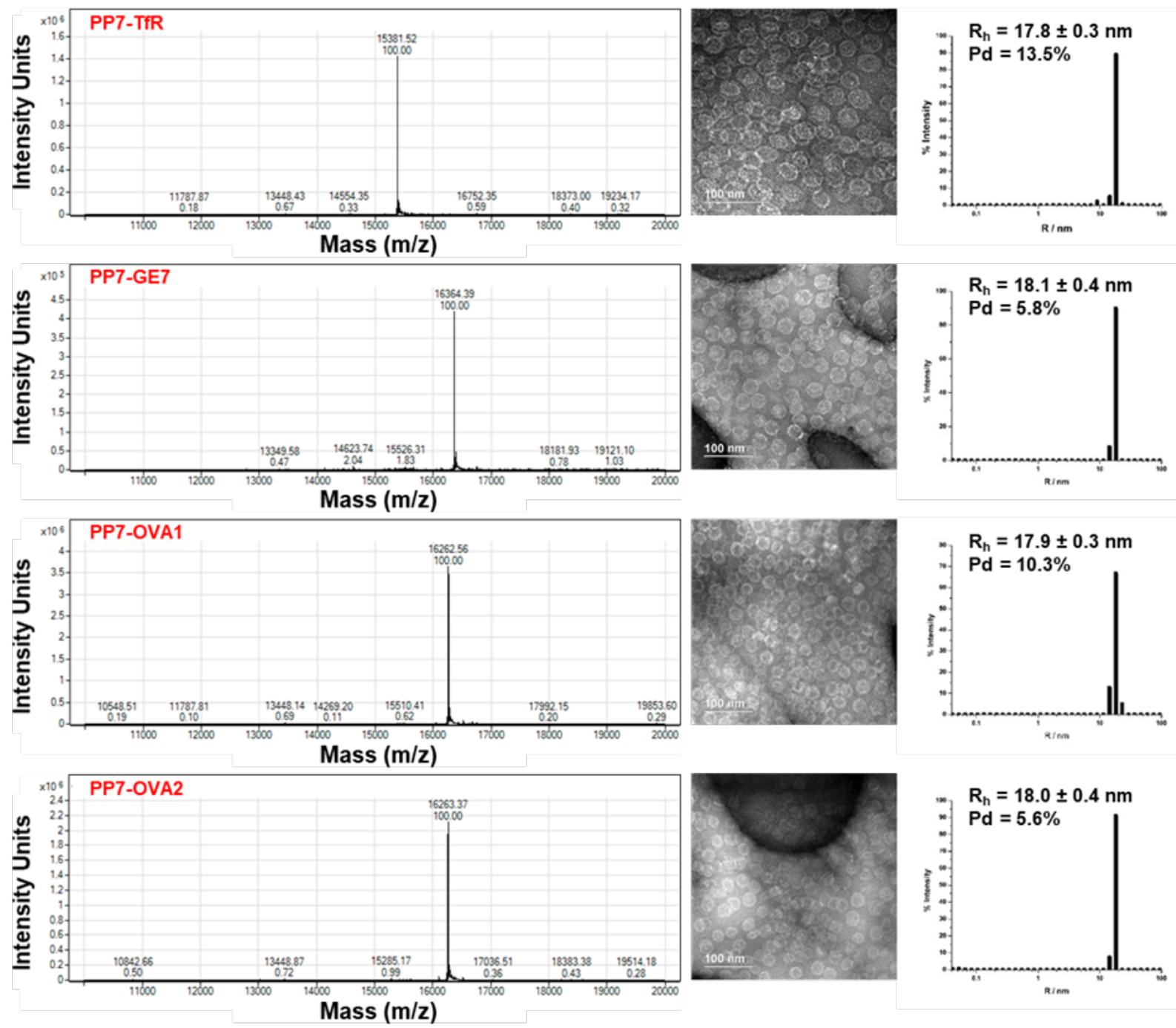
Figure S4. Characterization of the indicated proteins comprising homogeneous dimeric PP7 particles with other extensions by representative LC-MS (left), TEM of negative-stained (uranyl acetate) particles (middle), and DLS (right).
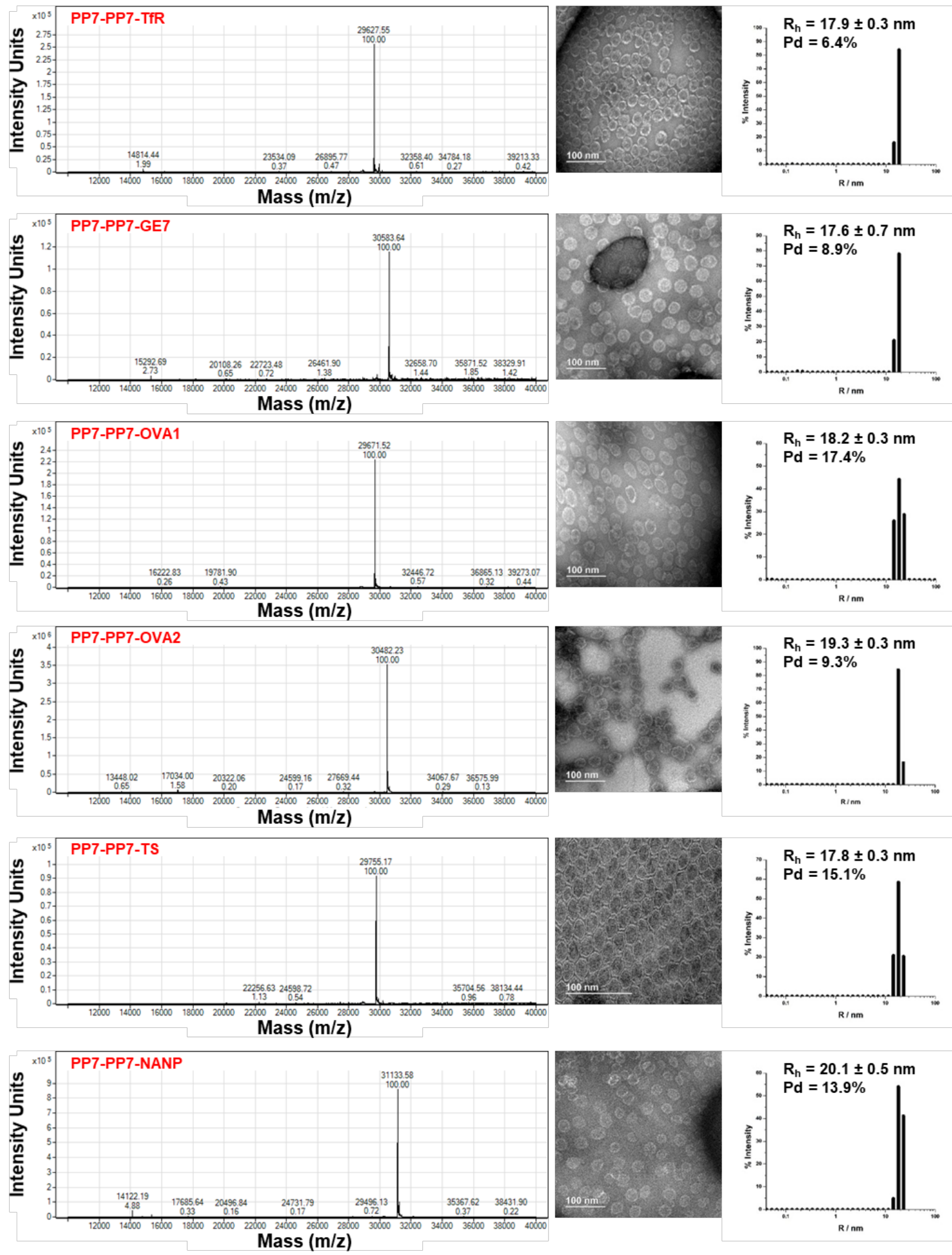
Figure S5. Characterization of the indicated proteins comprising homogeneous PP7 particles with loop insertions and C-terminal extensions by representative LC-MS (left), TEM of negative-stained (uranyl acetate) particles (middle), and DLS (right,).
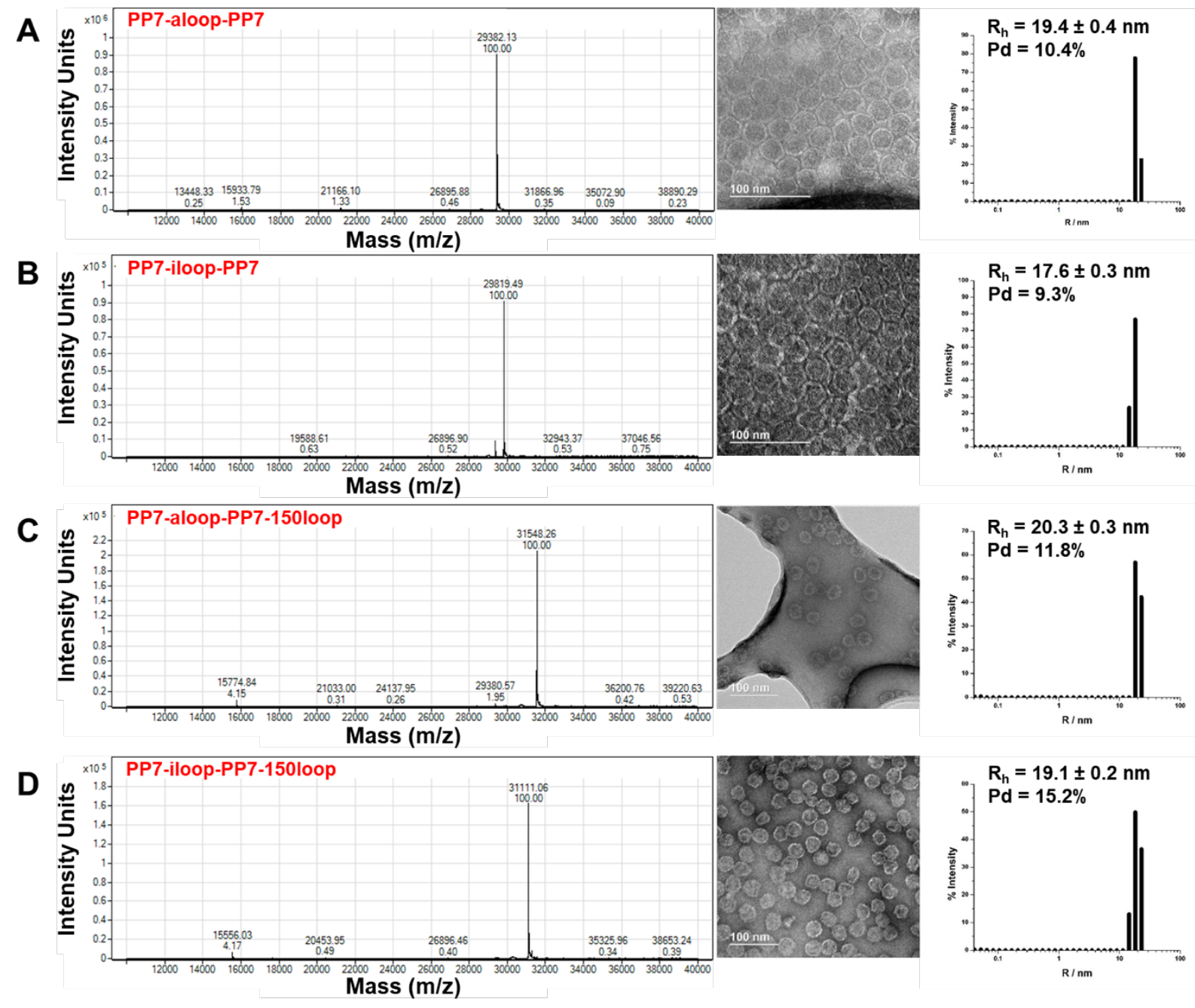
Figure S6. Analyses of thermal stability of the indicated PP7-PP7-based particles $(0.1 \mathrm{mg} / \mathrm{mL}$ in PBS buffer, heated at $10{ }^{\circ} \mathrm{C}$ per minute) in the absence and presence of $1 \mathrm{mM}$ dithiothreitol (DTT).
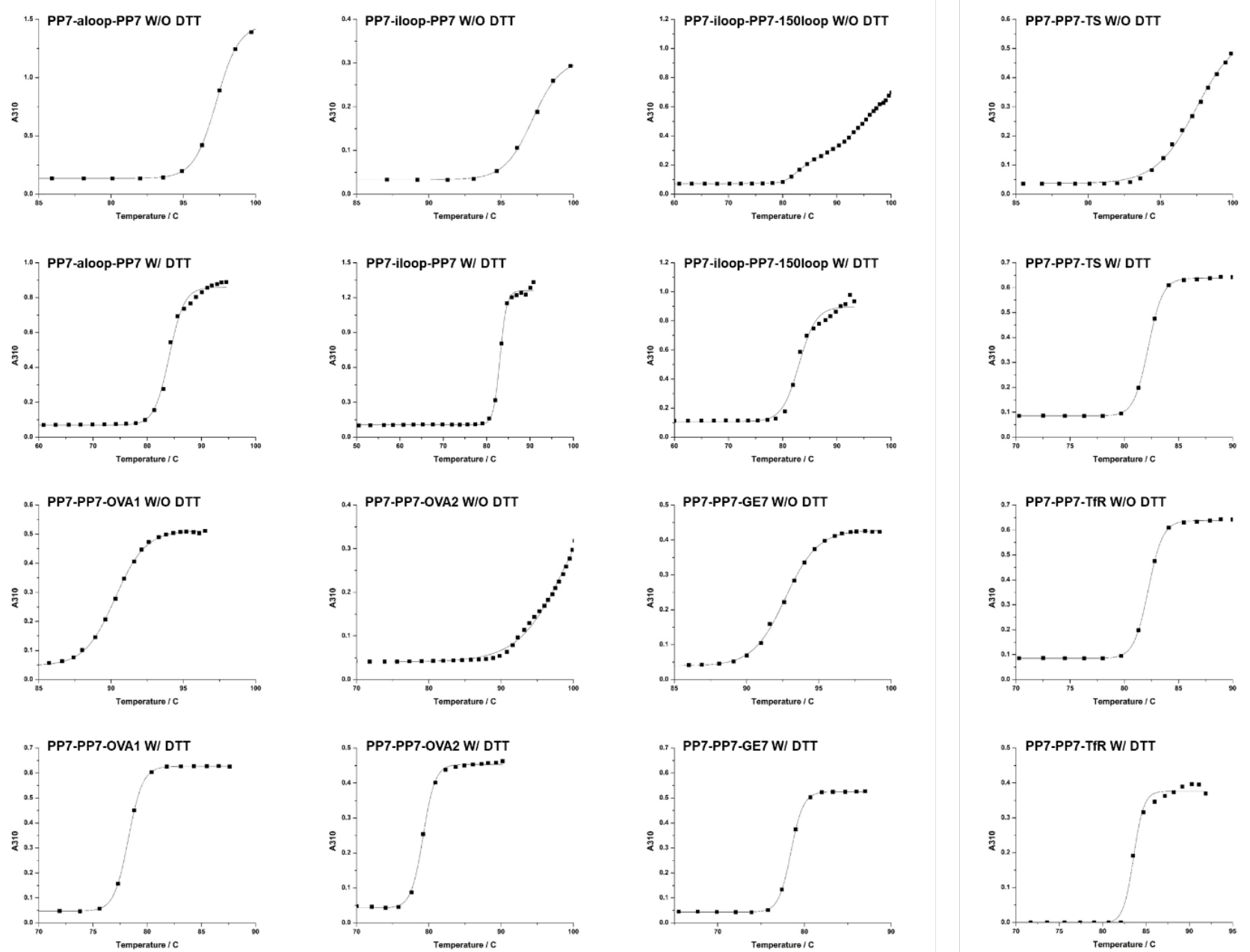
Figure S7. Cartoon representation of PP7 extension and loop insertion constructs. (A) monomeric PP7 with C-terminal extension; (B) dimeric PP7-PP7 with C-terminal extension; (C) dimeric PP7-PP7 with loop insertion; (D) dimeric PP7-PP7 with loop insertion and C-terminal extension.

(A) Monomeric PP7 presenting C-terminal extension

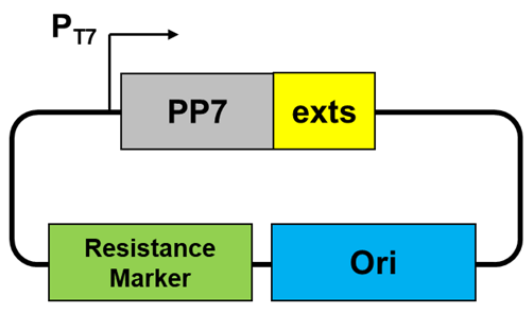

(B) Dimeric PP7 presenting C-terminal extension

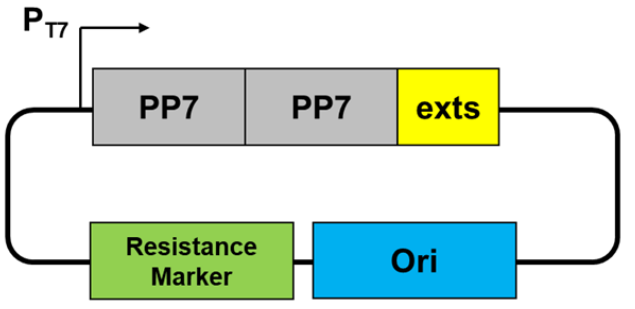

(C) Dimeric PP7 presenting loops in-between

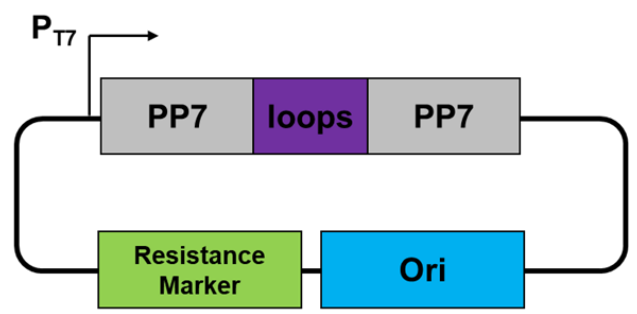

(D) Dimeric PP7 presenting loops in-between and C-terminal extension

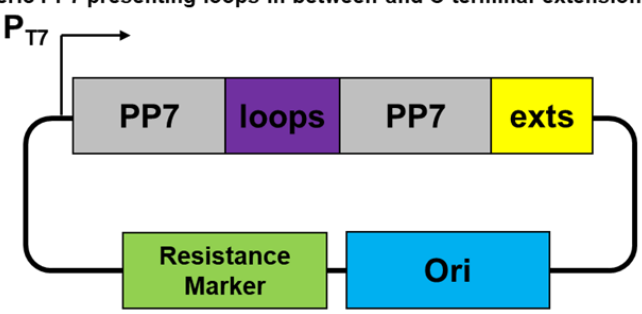

Figure S8. ZZ-PP7 reconstruction. (A) $10 \AA$ low -pass filtered ZZ-PP7 cryoEM map overlay with the 3.4 A ZZ-PP7 map PP7; (B) ZZ-PP7 low pass filtered map; (C) ZZ-PP7 low pass filtered map shown with the $T=3$ icosahedral cage (red). ZZ-PP7 image revealed displayed 60 regions of low-resolution electron density protruding outward with each representing three $\mathrm{N}$-terminal $\mathrm{ZZ}$ extensions, and therefore a total of 180 ZZ-domains presented per particle.

A

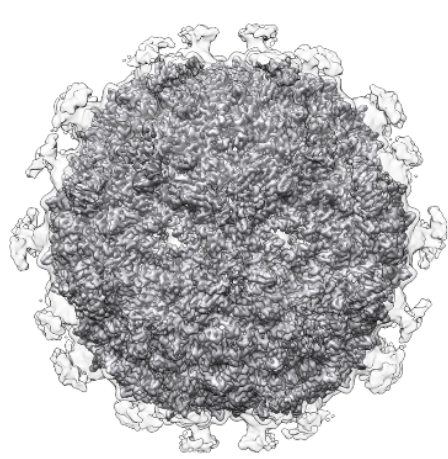

B

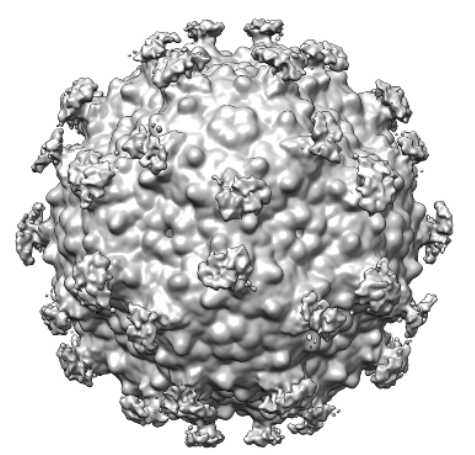

C

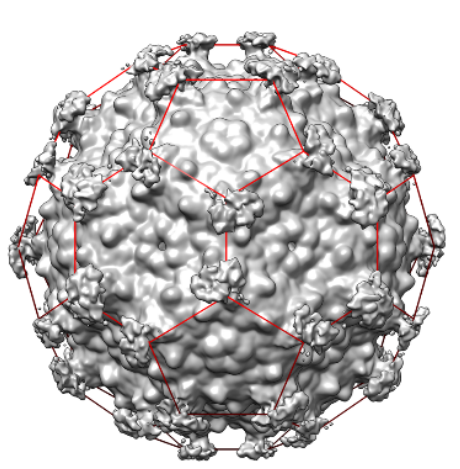

